International Journal of Information Technology \& Decision Making

Vol. 15, No. 6 (2016) 1579-1584

(C) World Scientific Publishing Company

DOI: $10.1142 /$ S0219622016990017

\title{
Author Index Volume 15 (2016)
}

Ai, S., Du, R., Brugha, C. M. and Wang, H., Pointing to priorities for multiple criteria decision making: The case of a MIS-based project in China

Aleskerov, F. and Petrushchenko, V., Dea by sequential exclusion of alternatives in heterogeneous samples

Alfares, H. K. and Duffuaa, S. O., Simulation-based evaluation of criteria rank-weighting methods in multi-criteria decision-making

Aliev, R. A., Huseynov, O. H. and Serdaroglu, R., Ranking of z-numbers and its application in decision making

Arenas, A. Y., see Barrios, M. A. O.

Back, A., see Silic, M.

Badagadze, O., see Sirbiladze, G.

Barfod, M. B., van den Honert, R. and Salling, K. B., Modeling group perceptions using stochastic simulation: Scaling issues in the multiplicative AHP

Barrios, M. A. O., De Felice, F., Negrete, K. P., Romero, B. A., Arenas, A. Y. and Petrillo, A., An AHP-topsis integrated model for selecting the most appropriate tomography equipment

Belacel, N., see He, S.

Bernardi, E. and Romagnoli, S., Distorted copula-based probability distribution of a counting hierarchical variable: A credit risk application

Boldt, M., see Borg, A.

Borg, A. and Boldt, M., Clustering residential burglaries using modus operandi and spatiotemporal information

Bouras, A., see Saksrisathaporn, K.

Bouslimani, Y., see He, S.

Brugha, C. M., see Ai, S.

Ceylan, R. and Koyuncu, H., A new breakpoint in hybrid particle swarm-neural network architecture: Individual boundary adjustment

Chan, A., see He, S.

Charles, A., see Saksrisathaporn, K.

Chen, J., see Zeng, S.

Chen, X., see Tan, C.

Chen, Z.-S., see Xiong, S.-H.

Cheng, C.-H., see Yeh, D.-Y.

15 (2016) 683-702

15 (2016) $5-22$

15 (2016) 43-61

15 (2016) 1503-1519

15 (2016) 861-885

15 (2016) 151-185

15 (2016) 1469-1502

15 (2016) 453-474

15 (2016) 861-885

15 (2016) 949-974

15 (2016) 285-310

15 (2016) 23-42

15 (2016) 23-42

15 (2016) 887-918

15 (2016) 949-974

15 (2016) 683-702

15 (2016) 1313-1343

15 (2016) 949-974

15 (2016) 887-918

15 (2016) 403-422

15 (2016) 311-352

15 (2016) 1055-1114

15 (2016) 187-213 
Cheng, M.-Y. and Hoang, N.-D., A self-adaptive fuzzy inference model based on least squares SVM for estimating compressive strength of rubberized concrete

Chin, K.-S., see Xiong, S.-H.

Chiu, J.-T. and Lin, C.-H., A modified particle swarm optimization based on eagle foraging behavior

Colak, I., see Kahraman, H. T.

Dai, Q., see Li, J.

De Falco, I., Scafuri, U. and Tarantino, E., Optimizing personalized touristic itineraries by a multiobjective evolutionary algorithm

De Felice, F., see Barrios, M. A. O.

De Maio, C., Tommasetti, A., Troisi, O., Vesci, M., Fenza, G. and Loia, V., Contextual fuzzy-based decision support system through opinion analysis: A case study at University of the Salerno

Domenech, J., Peña-Ortiz, R., Gil, J. A. and Pont, A., A methodology for economic evaluation of cloud-based web applications

Du, L., see Zhu, H.

Du, R., see Ai, S.

Duffuaa, S. O., see Alfares, H. K.

Eldemir, F. and Onden, I., Geographical information systems and multicriteria decisions integration approach for hospital location selection

Elouedi, Z., see Ennaceur, A.

Ennaceur, A., Elouedi, Z. and Lefevre, E., belief AHP method: AHP method with the Belief function framework

Farooq, U., Nongaillard, A., Ouzrout, Y. and Qadir, M. A., A feature-based reputation model for product evaluation

Fenza, G., see Maio, C. De

Fernandez-Ceniceros, J., see Martinez-De-Pison, E.

Ferreira, D. C. and Marques, R. C., Malmquist and Hicks-Moorsteen productivity indexes for clusters performance evaluation

Ferreira, F. A. F., see Jalali, M. S.

Ferreira, J. J. M., see Jalali, M. S.

Fotiadis, A., see Korol, T.

García, S., see Luna, F.

Garcia-Melon, M., see Romero-Gelvez, J. I.

Gil, J. A., see Domenech, J.

$\mathrm{Gu}$, J., see Ren, P.

Gu, W., Saaty, T. L. and Whitaker, R., Expert system for ice hockey game prediction: Data mining with human judgment

Hamam, H., see He, S.

He, S., Belacel, N., Chan, A., Hamam, H. and Bouslimani, Y., A hybrid artificial fish swarm simulated annealing optimization algorithm for automatic identification of clusters

Hoang, N.-D., see Cheng, M.-Y.

15 (2016) 603-619

15 (2016) 1055-1114

15 (2016) 703-727

15 (2016) 575-602

15 (2016) 1391-1421

15 (2016) 1269-1312

15 (2016) 861-885

15 (2016) 923-948

15 (2016) 1555-1578

15 (2016) 1345-1366

15 (2016) 683-702

15 (2016) 43-61

15 (2016) 975-997

15 (2016) 553-573

15 (2016) 553-573

15 (2016) 1521-1553

15 (2016) 923-948

15 (2016) 1211-1234

15 (2016) 1015-1053

15 (2016) 815-837

15 (2016) 815-837

15 (2016) 1451-1468

15 (2016) 479-515

15 (2016) 791-813

15 (2016) 1555-1578

15 (2016) 1367-1389

15 (2016) 763-789

15 (2016) 949-974

15 (2016) 949-974

15 (2016) 603-619 
Hsieh, Y.-H. and Yuan, S.-T., Can customer expectations be measured in real time?

15 (2016) 119-149

Huang, A., Qiao, H., Wang, S. and Liu, J., Improving forecasting performance by exploiting expert knowledge: Evidence from Guangzhou port

Huseynov, O. H., see Aliev, R. A.

Isasi, P., see Luna, F.

Jalali, M. S., Ferreira, F. A. F., Ferreira, J. J. M. and Meidute-Kavaliauskienè, I., Integrating metacognitive and psychometric decision-making approaches for bank customer loyalty measurement

Jusoh, A., see Zavadskas, E. K.

Kahraman, H. T., Sagiroglu, S. and Colak, I., Novel user modeling approaches for personalized learning environments

Kang, Z. and Morin, T., Multi-attribute decision making in a bidding game with imperfect information and uncertainty

Kapanadze, M., see Sirbiladze, G.

Kayvanfar, V., see Teymourian, E.

Khodarahmi, M., see Teymourian, E.

Khutsishvili, I., see Sirbiladze, G.

Komaki, Gh. M., see Teymourian, E.

Korol, T. and Fotiadis, A., Applying fuzzy logic of expert knowledge for accurate predictive algorithms of customer traffic flows in theme parks

Koyuncu, H., see Ceylan, R.

Lefevre, E., see Ennaceur, A.

Li, D., see Zeng, W.

Li, J., Li, M., Wu, D., Dai, Q. and Song, H., A Bayesian networksbased risk identification approach for software process risk: The context of chinese trustworthy software

Li, M., see Li, J.

$\mathrm{Li}, \mathrm{X}$., see Zeng, S.

Li, Y.-L., see Xiong, S.-H.

Lin, C.-H., see Chiu, J.-T.

Liu, J., see Huang, A.

Liu, P., Zhang, L., Liu, X. and Wang, P., Multi-valued neutrosophic number Bonferroni mean operators with their applications in multiple attribute group decision making

Liu, X., see Liu, P.

Liu, Y., Seah, H. S. and Shou, G., Cost-Optimal Time-dEpendent Routing with time and speed constraints in directed acyclic road networks

Loia, V., see Maio, C. De

Luna, F., Quintana, D., García, S. and Isasi, P., Enhancing financial portfolio robustness with an objective based on $\epsilon$-neighborhoods

Mardani, A., see Zavadskas, E. K.

Marques, R. C., see Ferreira, D. C.

15 (2016) 387-401

15 (2016) 1503-1519

15 (2016) 479-515
15 (2016) 1451-1468

15 (2016) 1313-1343

15 (2016) 553-573

15 (2016) 999-1014

15 (2016) 1391-1421

15 (2016) 1391-1421

15 (2016) 403-422

15 (2016) 1055-1114

15 (2016) 703-727

15 (2016) 387-401

15 (2016) 1181-1210

15 (2016) 1181-1210

15 (2016) 1413-1450

15 (2016) 923-948

15 (2016) 479-515

15 (2016) 645-682

15 (2016) 1015-1053 
Martinez-De-Pison, E., Fernandez-Ceniceros, J.,

Pernia-Espinoza, A. V., Martinez-De-Pison, F. J.

and Sanz-Garcia, A., Hotel reservation forecasting using flexible soft computing techniques: A case of study in a Spanish hotel

Martinez-De-Pison, F. J., see Martinez-De-Pison, E.

Meidutè-Kavaliauskienè, I., see Jalali, M. S.

Morin, T., see Kang, Z.

Naidu, J. V., see Thillaigovindan, N.

Negrete, K. P., see Barrios, M. A. O.

Nongaillard, A., see Farooq, U.

Nor, K. M., see Zavadskas, E. K.

Onden, I., see Eldemir, F.

Ouzrout, Y., see Farooq, U.

Ozcan, T. and Tuysuz, F., Modified grey relational analysis integrated with grey Dematel approach for the performance evaluation of retail stores

Peña-Ortiz, R., see Domenech, J.

Peng, J.-J., Wang, J.-Q. and Wu, X.-H., Novel multi-criteria decision-making approaches based on hesitant fuzzy sets and prospect theory

Pernia-Espinoza, A. V., see Martinez-De-Pison, E.

Petrillo, A., see Barrios, M. A. O.

Petrushchenko, V., see Aleskerov, F.

Phan, N., Poncelet, P. and Teisseire, M., All in one: Mining multiple movement patterns

Podvezko, V., see Zavadskas, E. K.

Poncelet, P., see Phan, N.

Pont, A., see Domenech, J.

Qadir, M. A., see Farooq, U.

Qiao, H., see Huang, A.

Quan, C. and Ren, F., Visualizing emotions from Chinese blogs by textual emotion analysis and recognition techniques

Quintana, D., see Luna, F.

Rajalaxmi, T. M., see Sujatha, R.

Reeveerakul, N., see Saksrisathaporn, K.

Ren, F., see Quan, C.

Ren, F., see Tang, Y.

Ren, P., Xu, Z. and Gu, J., Assessments of the effectiveness of an earthquake emergency plan implementation with hesitant analytic hierarchy process

Romagnoli, S., see Bernardi, E.

Romero-Gelvez, J. I. and Garcia-Melon, M., Influence analysis in consensus search - A multi criteria group decision making approach in environmental management

Romero, B. A., see Barrios, M. A. O.

Saaty, T. L. and Zhang, L., The need for adding judgment in Bayesian prediction

Saaty, T. L., see Gu, W.

Sagiroglu, S., see Kahraman, H. T.

15 (2016) 1211-1234

15 (2016) 1211-1234

15 (2016) 815-837

15 (2016) 63-81

15 (2016) 1157-1179

15 (2016) 861-885

15 (2016) 1521-1553

15 (2016) 645-682

15 (2016) 975-997

15 (2016) 1521-1553

15 (2016) 353-386

15 (2016) 1555-1578

15 (2016) 621-643

15 (2016) 1211-1234

15 (2016) 861-885

15 (2016) $5-22$

15 (2016) 1115-1156

15 (2016) 267-283

15 (2016) 1115-1156

15 (2016) 1555-1578

15 (2016) 1521-1553

15 (2016) 387-401

15 (2016) 215-234

15 (2016) 479-515

15 (2016) 83-118

15 (2016) 887-918

15 (2016) 215-234

15 (2016) 1235-1264

15 (2016) 1367-1389

15 (2016) 285-310

15 (2016) 791-813

15 (2016) 861-885

15 (2016) 733-761

15 (2016) 763-789

15 (2016) 575-602 
Saksrisathaporn, K., Bouras, A., Reeveerakul, N. and Charles, A., Application of a decision model by using an integration of AHP and topsis approaches within humanitarian operation life cycle

Salling, K. B., see Barfod, M. B.

Sanz-Garcia, A., see Martinez-De-Pison, E.

Scafuri, U., see De Falco, I.

Seah, H. S., see Liu, Y.

Serdaroglu, R., see Aliev, R. A.

Shanthi, S. A., see Thillaigovindan, N.

Shou, G., see Liu, Y.

Silic, M. and Back, A., The influence of risk factors in decision-making process for open source software adoption

Sirbiladze, G., Khutsishvili, I., Badagadze, O. and Kapanadze, M., More precise decision-making methodology in the temporalized body of evidence. Application in the information technology management

Sirbiladze, G., New fuzzy aggregation operators based on the finite choquet integral: Application in the MADM problem

Song, H., see $\mathrm{Li}$, J.

Sujatha, R. and Rajalaxmi, T. M., Hierarchical fuzzy hidden Markov chain for web applications

Tan, C. and Chen, X., Generalized Archimedean intuitionistic fuzzy averaging aggregation operators and their application to multicriteria decision-making

Tang, L., see Yu, L.

Tang, Y. and Ren, F., Variable differently implicational inference for R- and S-implications

Tarantino, E., see De Falco, I.

Teisseire, M., see Phan, N.

Teymourian, E., Kayvanfar, V., Komaki, Gh. M. and Khodarahmi, M., An enhanced intelligent water drops algorithm for scheduling of an agile manufacturing system

Thillaigovindan, N., Shanthi, S. A. and Naidu, J. V., New method for solving a general multiple criteria decision-making problem under risk in fuzzy environment

Tommasetti, A., see Maio, C. De

Troisi, O., see Maio, C. De

Turskis, Z., see Zavadskas, E. K.

Tuysuz, F., see Ozcan, T.

van den Honert, R., see Barfod, M. B.

Vargas, L. G., Voting with intensity of preferences

Vesci, M., see Maio, C. De

Wang, H., see Ai, S.

Wang, J.-Q., see Peng, J.-J.

Wang, P., see Liu, P.

Wang, P., see Zeng, W.

Wang, S., see Huang, A.

Whitaker, R., see $\mathrm{Gu}, \mathrm{W}$.

$\mathrm{Wu}$, D., see $\mathrm{Li}$, J.

Wu, X.-H., see Peng, J.-J.

15 (2016) 887-918

15 (2016) 453-474

15 (2016) 1211-1234

15 (2016) 1269-1312

15 (2016) 1413-1450

15 (2016) 1503-1519

15 (2016) 1157-1179

15 (2016) 1413-1450

15 (2016) 151-185

15 (2016) 1469-1502

15 (2016) 517-551

15 (2016) 1391-1421

15 (2016) 83-118

15 (2016) 311-352

15 (2016) 423-451

15 (2016) 1235-1264

15 (2016) 1269-1312

15 (2016) 1115-1156

15 (2016) 239-266

15 (2016) 1157-1179

15 (2016) 923-948

15 (2016) 923-948

15 (2016) 645-682

15 (2016) 353-386

15 (2016) 453-474

15 (2016) 839-859

15 (2016) 923-948

15 (2016) 683-702

15 (2016) 621-643

15 (2016) 1181-1210

15 (2016) 999-1014

15 (2016) 387-401

15 (2016) 763-789

15 (2016) 1391-1421

15 (2016) 621-643 
Xiong, S.-H., Chen, Z.-S., Li, Y.-L. and Chin, K.-S., On extending power-geometric operators to interval-valued hesitant fuzzy sets and their applications to group decision making

15 (2016) 1055-1114

$\mathrm{Xu}, \mathrm{Y}$., see Zhu, H.

15 (2016) 1345-1366

$\mathrm{Xu}, \mathrm{Z}$., see Ren, $\mathrm{P}$.

Yang, Z., see Yu, L.

Yeh, D.-Y. and Cheng, C.-H., Performance management of Taiwan's national hospitals

Yu, L., Yang, Z. and Tang, L., Prediction-based multi-objective optimization for oil purchasing and distribution with the NSGA-II algorithm

15 (2016) $1367-1389$

15 (2016) 423-451

15 (2016) $187-213$

15 (2016) 423-451

15 (2016) 119-149

Yuan, S.-T., see Hsieh, Y.-H.

Zavadskas, E. K. and Podvezko, V., Integrated determination of objective criteria weights in MCDM

15 (2016) 267-283

Zavadskas, E. K., Mardani, A., Turskis, Z., Jusoh, A. and Nor, K. M., Development of TOPSIS method to solve complicated decision-making problems: An overview on developments from 2000 to 2015

Zeng, S., Chen, J. and Li, X., A hybrid method for Pythagorean fuzzy multiple-criteria decision making

Zeng, W., Li, D. and Wang, P., Variable weight decision making and balance function analysis based on factor space

Zhang, L., see Liu, P.

Zhang, L., see Saaty, T. L.

Zhao, J., see Zhu, H.

Zhu, H., Zhao, J., Xu, Y. and Du, L., Interval-valued belief rule inference methodology based on evidential reasoning-irimer

15 (2016) 645-682

15 (2016) 403-422

15 (2016) 999-1014

15 (2016) 1181-1210

15 (2016) 733-761

15 (2016) 1345-1366

15 (2016) 1345-1366 